\title{
MRI VERSUS ARTHROSCOPY: A COMPARISON OF FINDINGS
}

\author{
Somashekhara Reddy Pereddy ${ }^{1}$
}

${ }^{1}$ Associate Professor, Department of Orthopaedics, Apollo Institute of Medical Sciences and Research Centre.

\begin{abstract}
The purpose of this study is to correlate the findings of Magnetic Resonance Imaging with Arthroscopy in internal derangements of knee and to assess the sensitivity, specificity and accuracy of Magnetic Resonance Imaging in comparison to Arthroscopy.
\end{abstract}

\section{METHODS AND MATERIALS}

Fifty six patients with history of suspected internal derangement of knee were evaluated prospectively with MRI and correlated with arthroscopy during a one and half a year period from March 2012 to September 2013.1.5 Tesla MRI machine using a closed extremity coil is used. Sequences used in MRI of the knee include Turbo-spin echo (TSE), FFE (Gradient echo) and Short Tau Inversion Recovery (STIR) sequences in Axial, Sagittal and Coronal planes using a slice thickness of $3 \mathrm{~mm}$ with a $0.3 \mathrm{~mm}$ slice gap. Arthroscopy of the knee with standard anteromedial and anterolateral portals with the patient in supine position done in all the cases.

\section{RESULTS}

The diagnostic sensitivity, specificity and accuracy of MRI in detecting cruciate ligament and meniscal injuries as compared to Arthroscopy are as follows: Anterior Cruciate Ligament (ACL): 97.29\%, 89.47\%, 94.64\%; Posterior Cruciate Ligament (PCL): 100\%, 100\%, 100\%; Medial Meniscus (MM): 100\%, 93.33\%, 98.21\% and Lateral Meniscus (LM): 93.10, 92.59, 92.85. Most common lesions found were ACL and Medial Meniscal tears (posterior horn of MM being the most common site).

\section{SUMMARY AND CONCLUSION}

- MRI is a useful non-invasive modality having high sensitivity, specificity and accuracy in diagnosing cruciate ligament and meniscal injuries.

- $\quad$ MRI should be considered as the first line of investigation in all patients with suspected internal derangements of knee.

- MRI being easily available and non-invasive is useful as a pre-operative screening modality, thus improves the quality of diagnostic and therapeutic arthroscopies and further reduces the morbidity.

- Arthroscopy is the standard diagnosing tool in all patients with suspected internal derangements of knee.

- Arthroscopy should be reserved for selected cases where intervention is required and as a problem solving tool.

\section{KEYWORDS}

MRI, Arthroscopy.

HOW TO CITE THIS ARTICLE: Pereddy SR. MRI versus arthroscopy: a comparison of findings. J. Evolution Med. Dent. Sci. 2016;5(49):3149-3154, DOI: 10.14260/jemds/2016/731

\section{INTRODUCTION}

The knee joint is one of the most vulnerable and frequently injured joints of the body. The injuries can be either acute or chronic and they constitute a major cause of pain and instability.

The various modalities currently used to evaluate pathological conditions of the knee include Conventional Radiography, Fluoroscopy, Arthrography, Sonography, Computed Tomography, Nuclear Medicine, Magnetic Resonance Imaging and Arthroscopy.

The imaging of most pathological entities requires plain films. The use of Fluoroscopy and Sonography to guide interventional procedures and $\mathrm{CT}$ to evaluate complex fractures has become routine. The role of MRI in imaging of knee has steadily increased over the years and is often the main or only imaging tool for evaluation of suspected internal derangements of knee. ${ }^{1}$

Financial or Other, Competing Interest: None.

Submission 28-03-2016, Peer Review 26-05-2016,

Acceptance 02-06-2016, Published 18-06-2016.

Corresponding Author:

Dr. Somashekhara Reddy Pereddy,

Villa 90, Villa Scapes, Gandipet,

K. V. Ranga Reddy Dist., Hyderabad,

Telangana.

E-mail: drpereddysr@gmail.com

DOI: $10.14260 /$ jemds/2016/731
The most significant advances in imaging of the knee has been made in the realm of Magnetic Resonance Imaging, which has clearly emerged as a primary tool in evaluation and guiding the management of internal derangements of knee. With the development of newer sequences with improved SNR, higher resolution, shorter imaging times and improved accuracy, MRI has changed the traditional algorithm for workup of suspected internal derangements of knee.

Advantages of MRI over other imaging modalities include lack of ionizing radiation, excellent soft tissue contrast, multiplanar imaging capabilities, non-invasive and do not require manipulation of the knee as in Arthrography.

MRI Cartigram is showing promise in evaluation of cartilage lesions of the knee, which is now being increasingly done in many centres.

\footnotetext{
Arthroscopy

During an average knee arthroscopy, a small fiberoptic camera (the Arthroscope) is inserted into the joint through a small incision, about $4 \mathrm{~mm}(1 / 8 \mathrm{inch})$ long. A special fluid is used to visualize the joint parts. More incisions might be performed in order to check other parts of the knee. Then other miniature instruments are used and the surgery is performed.
} 


\section{AIMS AND OBJECTIVES}

1. To correlate the findings of Magnetic Resonance Imaging with Arthroscopy in internal derangements of Knee.

2. To assess the sensitivity, specificity and accuracy of Magnetic Resonance Imaging in comparison to Arthroscopy.

\section{REVIEW OF LITERATURE}

The potential of MRI in the evaluation of knee was explored as early as 1983 by Kean et $\mathrm{al}^{2}$ Moon and Associates. ${ }^{3}$ and also subsequently by $\mathrm{Li}$ et $\mathrm{al}^{4}$ in 1984 . However, these studies had limitations, as the spatial resolution was poor due to lack of dedicated surface coils.

In 1987, Hajek and Baker. ${ }^{5}$ proposed intra-articular contrast media might enhance the diagnostic capabilities of MR imaging in the setting of joint pathology.

Recently, it has been discovered that the Danish physician Severin Nordentoft reported on arthroscopies of the knee joint as early as 1912 at the Proceedings of the 41st Congress of the German Society of Surgeons at Berlin. ${ }^{6}$

Pioneering work in the field of arthroscopy began as early as the 1920s with the work of Eugen Bircher. ${ }^{7}$ Bircher published several papers in the 1920 s about his use of arthroscopy of the knee for diagnostic purposes. ${ }^{7}$ After diagnosing torn tissue through arthroscopy, Bircher used open surgery to remove or repair the damaged tissue.

Ruwe and Collaborators. ${ }^{8}$ in a study addressing the issue of cost effectiveness indicated that the results of MRI in 53 of 103 patients avoided a potentially unnecessary diagnostic arthroscopy.

Elvenes et $\mathrm{al}^{9}$ in their study found that sensitivity, specificity, positive and negative predictive values of MRI for medial meniscus were $100 \%, 77 \%, 71 \%$ and $100 \%$ respectively, while values for lateral meniscus were $40 \%$, 89\%, 33\% and 91\% respectively. Overall accuracy of MRI for medial meniscus and lateral meniscus combined was $84 \%$. On basis of high negative value, they concluded that MRI is useful to exclude patients from unnecessary arthroscopy.

Pappenport et al ${ }^{10}$ in their study "MR imaging before arthroscopy in knee disorders" reviewed current literature and concluded that MR examination of the knee should be performed before arthroscopy is undertaken. Meniscal tears can be detected with accuracy rates of $90 \%$ and ACL tears with 93\% compared with arthroscopy. However, arthroscopy is not the gold standard since it has weak points, viz. peripheral meniscal tears, osteochondritis without apparent damage to the cartilage.

Kaplan et al ${ }^{11}$ concluded from their study that contusions involving the posterior medial tibial plateau may result from contrecoup injuries directly following ACL tears as the knee reduces. These are almost always associated with a far more peripheral meniscal tear/with a menisco-capsular junction injury affecting the posterior horn of medial meniscus.

Arndt William et $\mathrm{al}^{12}$ found that because of their comparable accuracy and short imaging times, Coronal FSE with Fat saturation and FSE STIR sequences are superior to conventional STIR sequences for diagnosis of bone contusions in the knee.

Sonin et $\mathrm{al}^{13}$ found that injuries of the PCL occur less often than injuries to ACL and makes up to $2-23 \%$ of all knee injuries. In $30 \%$ of these cases, PCL injury is isolated. In the rest, there is coexistent ligament injury. They concluded that
MRI is accurate in assessment of PCL injuries. Sagittal MR images are best because most occur in mid substance of PCL, which is not seen well on coronal/axial images. Normal appears as continuous band of low signal intensity on all sequences throughout its arcuate course.

Schweitzer et al14 in 1995 evaluated multiple potential signs of MCL injuries and accuracy of previously described criteria used to grade MCL injuries in 76 patients with MCL sprain. They concluded that fascial oedema and loss of demarcation from adjacent fat to be most sensitive signs and MR imaging grading system might not be accurate for classification of MCL injuries.

Lateral collateral ligament extends from lateral femoral condyle to fibular head. When LCL is disrupted, it is common to see associated tears in PCL. Secondary findings such as joint space widening, effusion, meniscal tears, cruciate ligament tears and bone bruise are not unusual. Yao et $\mathrm{al}^{15}$ reported $87 \%$ accuracy for MRI in classifying LCL injuries. Partial or grade 2 tears are more difficult to correctly classify with MRI.

Levinson et al ${ }^{16}$ from their study found that the accuracy of arthroscopy in identification of inferior surface tears of posterior horn of medial meniscus is as low as 45 to $65 \%$. Further arthrographic and arthroscopic surface evaluation are insensitive to Grade I and Grade II intrasubstance degeneration as precursors to the formation of a defined meniscal tear as described by Raunest et al. ${ }^{17}$ MRI also detects multiple meniscal tears that may be overlooked on Arthrography.

Arthur De Smet et al $^{18}$ studied 200 patients with 108 medial and 58 lateral meniscal tears. They found that menisci with signal intensity possibly contacting the surface had the same frequency of the tear as menisci without signal contacting the surface. More than $90 \%$ of menisci with signal contacting the surface on more than one image were torn, but only $55 \%$ of medial and $30 \%$ lateral menisci with such signal on only one image were torn. So the authors concluded that a tear is less likely if internal signal in contact with the surface was present in only one image.

\section{MATERIALS AND METHODS}

Fifty six patients with history of suspected internal derangements of knee were evaluated prospectively with MRI and correlated with Arthroscopy during a one and a half year period between March 2012 and September 2013. MR imaging was performed on a 1.5 Tesla (Philips Achieva) machine. Relevant clinical history and clinical findings were taken into consideration in performing MRI. In this study, arthroscopy was considered to be the gold standard and MRI findings were correlated.

\section{Imaging Protocol and Pulse Sequences}

Routinely used sequences in MR imaging of the knee include Turbo-Spin Echo (TSE), FFE (Gradient echo) and Short Tau Inversion Recovery (STIR) sequences. The standard imaging planes used are Axial, Sagittal and Coronal views. Imaging protocols for MRI knee was done using a Field Of View (FOV) of $200 \times 200 \mathrm{~mm}, 256 \times 256$ matrix and slice thickness of 3 $\mathrm{mm}$ with a $0.3 \mathrm{~mm}$ gap.

An axial acquisition through the patella-femoral joint is used as an initial localiser for subsequent sagittal and coronal imaging planes. 


\section{ARTHROSCOPY}

Arthroscopy was performed within a period of approximately 1 day to 20 days. Arthroscopy was performed by senior Orthopaedicians. The MR images were reported by Senior Radiologists. In our study, the orthopaedic surgeon performing the arthroscopy was aware of the MRI findings. Patients with grade 1 and grade 2 meniscal tears were excluded from the study, as these lesions do not reach the articular surface and are not visualised during arthroscopy. The images were reviewed for the presence of ACL, PCL and/or meniscal tears.

ACL and PCL tears were diagnosed based on the presence of any of the primary signs. Only grade 3 meniscal tears, which extend to one of the articular surfaces were included in the study.

The sensitivity, specificity and accuracy of MRI was based on the findings of arthroscopy, as arthroscopy was taken as the gold standard for the diagnosis of internal derangements of the knee.

\section{IMAGES}

Case 5

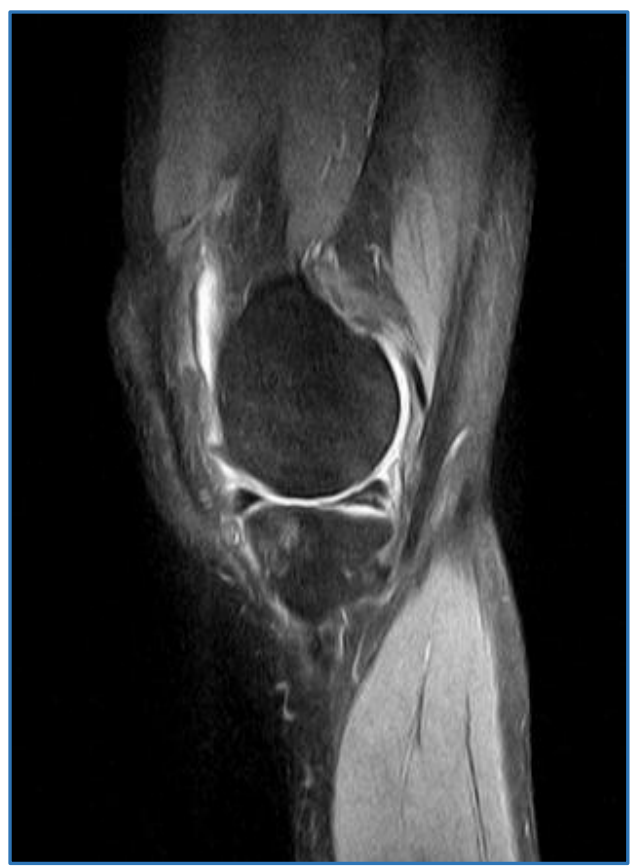

Posterior Horn Tear of Medial Meniscus on PDW Sag Image

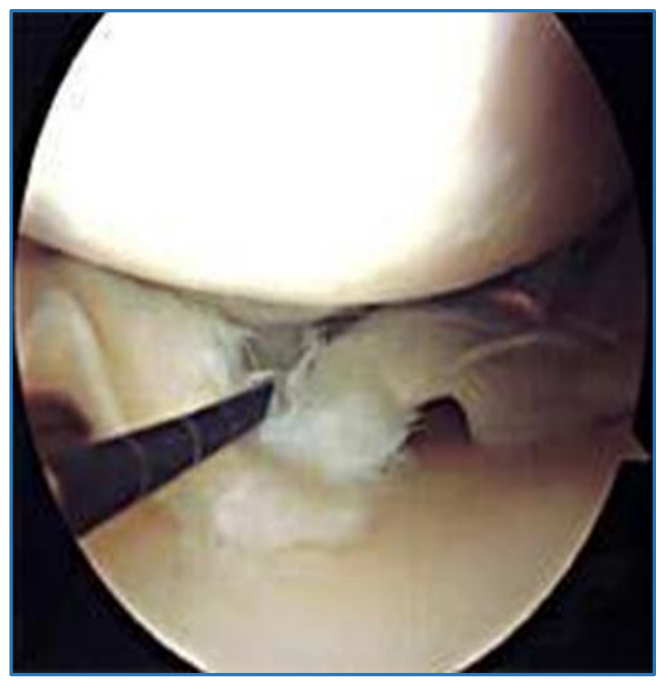

Medial Meniscal Tear on Arthroscopy
Case 23

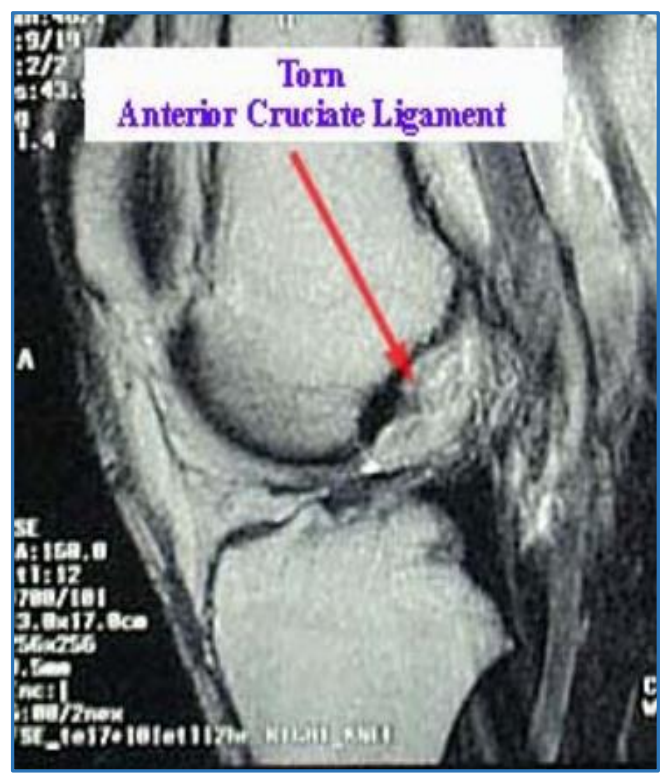

ACL Tear on PDW Sag Image

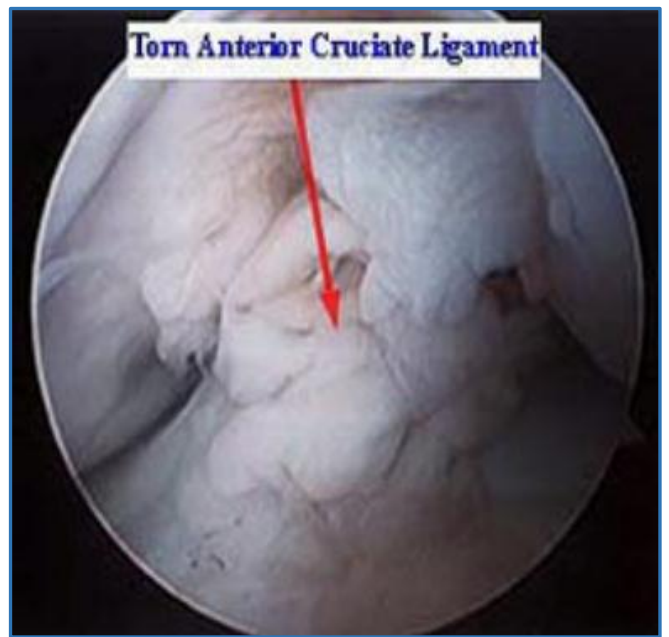

ACL Tear on Arthroscopy

\section{CASE 10}

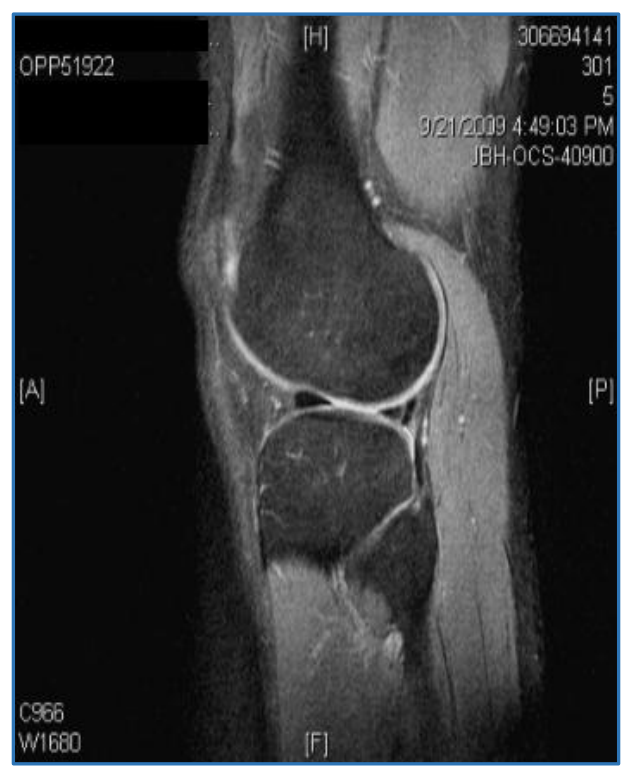

Posterior Horn Tear of Lateral Meniscus on PDW Sagittal Image 


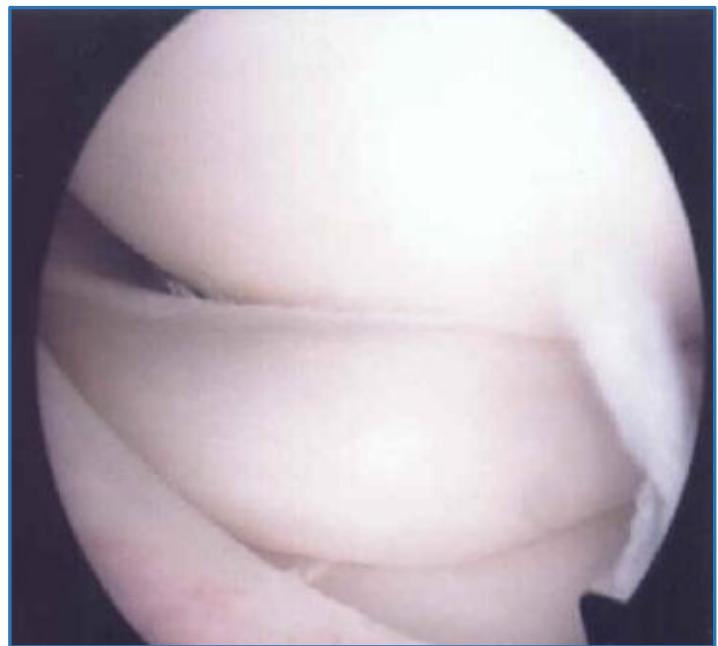

Lateral Meniscal Tear on Arthroscopy

Case 3

MRI - PCL tear+Anterior horn tear of lateral meniscus. Arthroscopy - PCL avulsion and lax ACL.

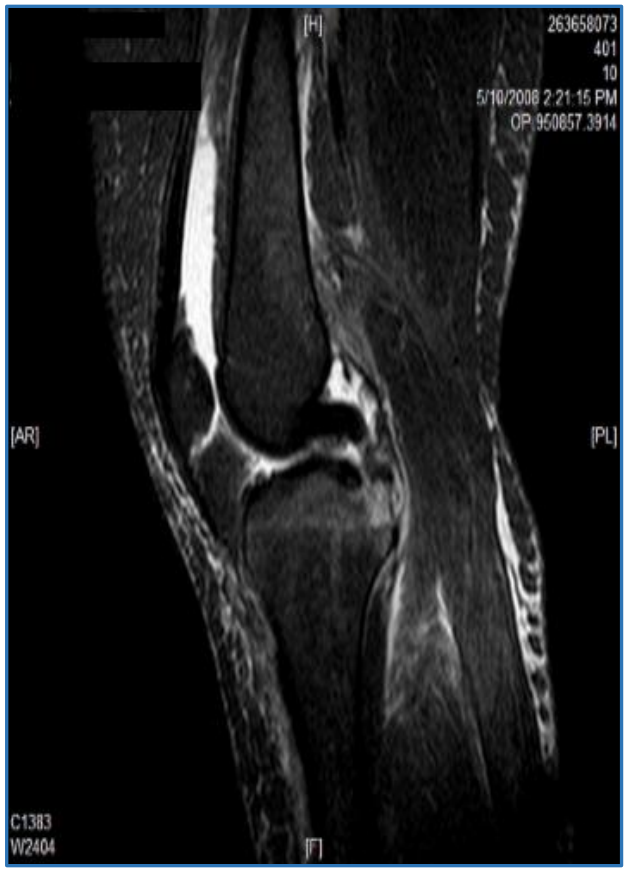

PCL Tear at Tibial Insertion on Stir Sagittal Image

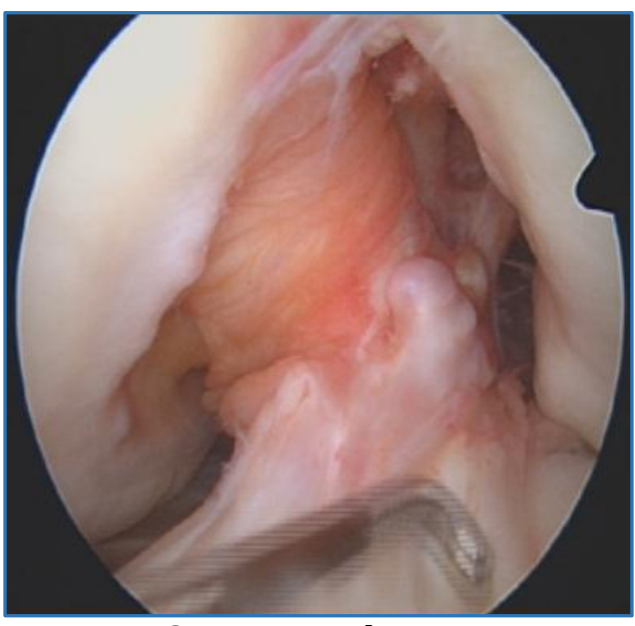

PCL Tear on Arthroscopy
MRI - Posterior horn tear of medial meniscus.

Arthroscopy - Bucket-handle tear of medial meniscus.

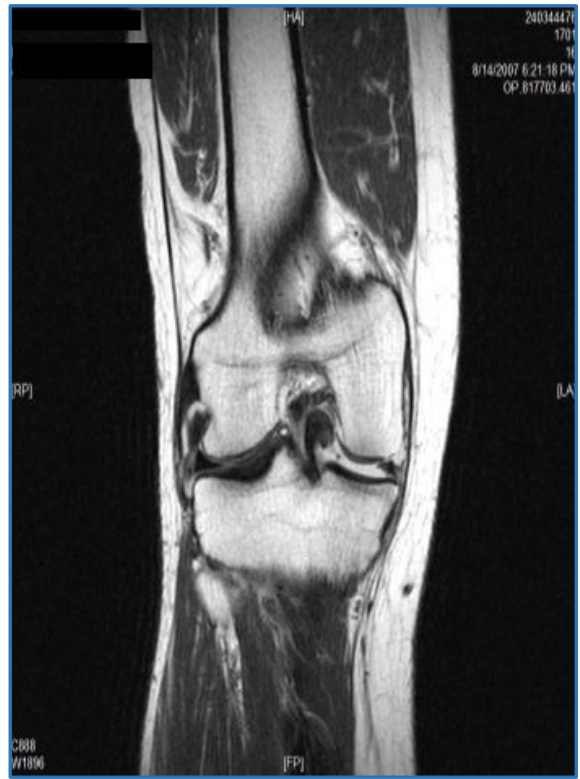

Bucket Handle Tear of Medial Meniscus

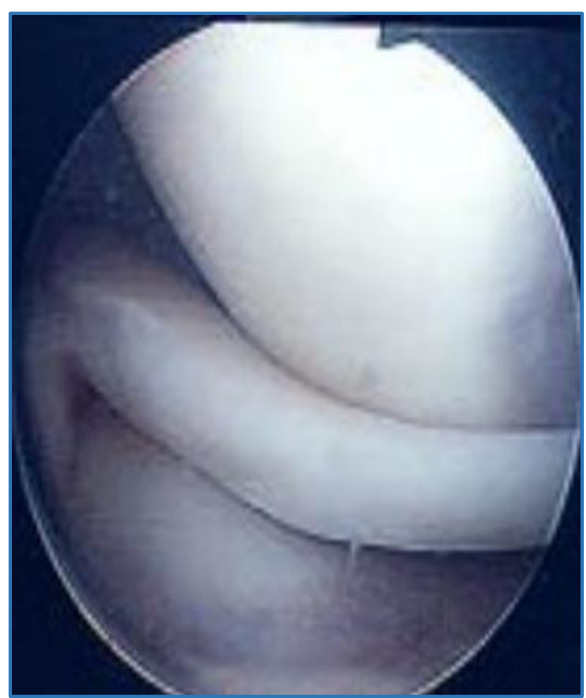

Bucket Handle Tear on Arthroscopy

\section{OBSERVATIONS}

A total of 56 patients in suspected internal derangements of knee were evaluated with MRI and subsequently by Arthroscopy. Out of 56 patients, 44 were males and 12 were females. Patients age range from 14 to 65 years (Mean of 35 yrs.). Patients in the age group of 21-30 years accounted for maximum number of cases.

\begin{tabular}{|c|c|c|}
\hline & MRI & Arthroscopy \\
\hline ACL & 38 & 37 \\
\hline PCL & 3 & 3 \\
\hline Medial Meniscus & 42 & 41 \\
\hline Lateral Meniscus & 29 & 29 \\
\hline
\end{tabular}

Case 38 


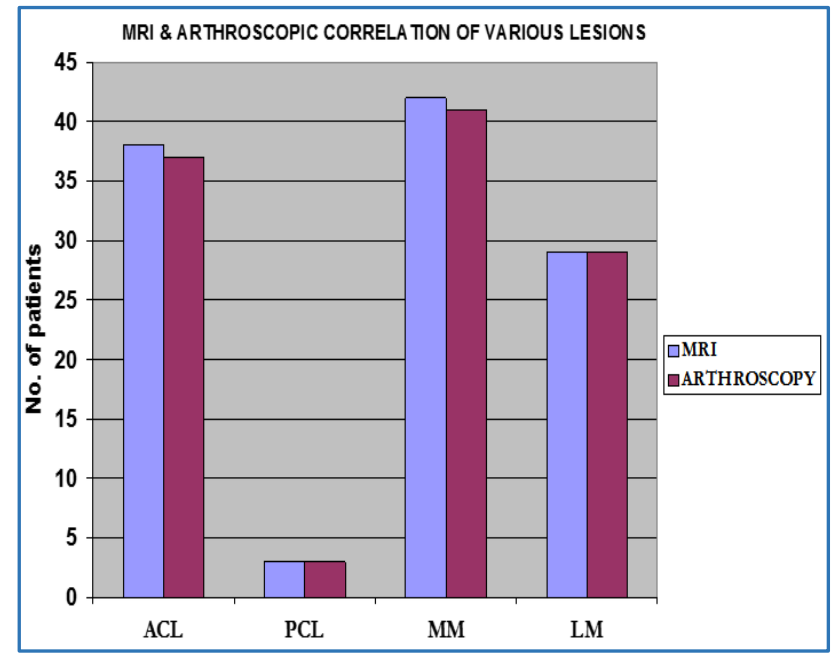

\section{Cruciate Ligament Tears}

Out of 56 patients, 38 cases showed ACL tears and 3 cases showed PCL tears. ACL tears were also associated with tears like meniscal tears and PCL tears; 28 out of 38 ACL tears showed associated meniscal tears. Buckling of PCL was seen in 7 cases of ACL tears. Isolated ACL tears were seen in 8 cases. Of the 3 cases of PCL tears, 2 cases were associated with ACL tears also.

MRI findings of ACL tears were correlated with Arthroscopy. Sensitivity, specificity and accuracy of MRI in ACL tears were $97.29 \%, 89.47 \%$ and $94.64 \%$ respectively.

All the 3 patients who had PCL tears on MRI were reported to have PCL tears on Arthroscopy. The diagnostic sensitivity, specificity and accuracy of MRI in diagnosing PCL tears are $100 \%$ in this study.

\section{Meniscal Tears}

Out of 56 patients, 42 cases showed medial meniscal tears and 29 cases showed lateral meniscal tears; 27 cases of medial meniscal tears were associated with ACL tears and 18 cases of lateral meniscal tears were associated with ACL tears. Both meniscal tears were seen in 24 cases.

5 cases of bucket handle tears were seen in medial meniscus.

Discoid meniscus with tear was seen in one patient involving medial meniscus.

Out of 42 patients with medial meniscal tears, 3 cases showed tears in anterior horn, 7 cases showed tears in the body and 41 cases showed tears in the posterior horn.

Out of 29 patients with lateral meniscal tears anterior horn tears were seen in 19 cases, tears in the body were seen in 2 cases and posterior horn tears were seen in 17 cases.

All the patients who had grade III meniscal tears were correlated with Arthroscopy. Patients with grade I and grade II tears seen on MRI were excluded from the study, as the correlation was with Arthroscopy, where only grade III tears are visualized.

The sensitivity, specificity and accuracy of MRI in diagnosing medial meniscal tears were 100\%, 93.33\% and 98.25\% respectively when correlated with Arthroscopy.

The diagnostic sensitivity, specificity and accuracy of MRI for lateral meniscal tears were $93.10 \%, 92.59 \%$ and $92.85 \%$ respectively when correlated with Arthroscopy.

\begin{tabular}{|c|c|c|c|c|}
\hline & ACL & PCL & $\begin{array}{c}\text { Medial } \\
\text { Meniscus }\end{array}$ & $\begin{array}{c}\text { Lateral } \\
\text { Meniscus }\end{array}$ \\
\hline True Positives & 36 & 3 & 41 & 27 \\
\hline False Positives & 2 & 0 & 1 & 2 \\
\hline $\begin{array}{c}\text { False } \\
\text { Negatives }\end{array}$ & 1 & 0 & 0 & 2 \\
\hline True Negatives & 17 & 53 & 14 & 25 \\
\hline Sensitivity & 97.29 & 100 & 100 & 93.10 \\
\hline Specificity & 89.47 & 100 & 93.33 & 92.59 \\
\hline $\begin{array}{c}\text { Positive } \\
\text { Predictive } \\
\text { Value }\end{array}$ & 94.73 & 100 & 97.61 & 93.10 \\
\hline $\begin{array}{c}\text { Negative } \\
\text { Predictive } \\
\text { Value }\end{array}$ & 94.44 & 100 & 100 & 92.59 \\
\hline Accuracy & 94.64 & 100 & 98.21 & 92.85 \\
\hline
\end{tabular}

\section{DISCUSSION}

Our study spanned over a 11/2 year period from March 2010 to September 2011. Prospective evaluation of 56 patients with suspected of internal derangements of knee with MRI and subsequently Arthroscopy was done.

The cruciate ligaments and menisci were studied on both the modalities and comparisons were drawn.

Arthroscopy was considered as gold standard and the sensitivity, specificity and accuracy of MRI were calculated.

Of the 56 patients studied there were 38 ACL tears, 3 PCL tears, 42 medial meniscal tears and 29 lateral meniscal tears on MRI.

\section{Cruciate Ligament Tears}

The accuracy of MR imaging in assessing cruciate ligament tears has been reported to be high. MRI diagnosis of ACL tears was based on primary and secondary signs as described earlier. Out of 56 patients, 38 ACL tears and 3 PCL tears were seen.

Out of 38 ACL tears on MRI, 2 cases were reported to be normal ACLs on Arthroscopy (false positive). Rest of the 36 cases were confirmed on Arthroscopy. One case which was reported to have an ACL tear on Arthroscopy was reported as normal on MRI (false negative).

Two false positive cases of ACL tears were seen. One was because of intrasubstance hyperintensities, which was reported as normal on Arthroscopy. The other case showed non-visualization of ACL, which may be contributed to ligament laxity.

One false negative case of ACL tear was identified, which probably is because of a partial tear.

In evaluating ACL tears, the sensitivity, specificity and accuracy in our study were $97.29 \%, 89.47 \%$ and $94.64 \%$ respectively.

The role of MRI in diagnosing PCL tears is extremely accurate. In our study 3 PCL tears were seen, which were confirmed on Arthroscopy. The sensitivity, specificity and accuracy of MRI in detecting PCL tears in this study was $100 \%$.

\section{Meniscal Tears}

Role of MRI in assessing meniscal tears is extremely accurate. Patients with Grade III meniscal tears were only included in the study as Grade I and Grade II tears are not clearly visualized on Arthroscopy. Out of 56 patients, 42 cases had 
Grade III medial meniscal tears and 29 cases had Grade III lateral meniscal tears. Both meniscal tears were seen in 24 cases.

In our study, medial meniscal tears were more common than lateral meniscal tears. In our study, out of 42 medial meniscal tears, the common location was in the posterior horn. This was corresponding to the literature on medial meniscal tears.

Out of 42 medial meniscal tears on MRI, 41 tears were confirmed on Arthroscopy. One case which was reported to have a tear on MRI was not reported on Arthroscopy (false positive). This can be attributed to closed intrasubstance tear.

Five cases of Bucket handle tears of medial meniscus were identified, which were confirmed on Arthroscopy. Bucket handle tears of medial meniscus are more common than lateral meniscus.

Out of 29 lateral meniscal tears on MRI, 27 were confirmed on Arthroscopy. Two cases which were reported to have tears on MRI were not reported on Arthroscopy (false positive). Two cases reported to have tears on Arthroscopy could not be made out on MRI (false negative). These findings can be attributed to intrasubstance tears or small peripheral tears.

The sensitivity, specificity and accuracy of MRI in detecting Grade III tears of medial meniscus were $100 \%$, 93.33\% and $98.21 \%$ respectively and Grade III tears of lateral meniscus were $93.10 \%, 92.59 \%$ and $92.85 \%$ respectively.

\section{SUMMARY AND CONCLUSIONS}

MRI is a useful non-invasive modality having high sensitivity, specificity and accuracy in diagnosing cruciate ligament and meniscal injuries.

MRI is extremely useful in the evaluation of internal morphology and also the surface of the meniscus. MRI is more sensitive than Arthroscopy in detecting intrasubstance tears and small peripheral meniscal tears.

- MRI should be considered as the first line of investigation in all patients with suspected internal derangements of knee.

- Arthroscopy is the standard diagnosing tool in all patients with suspected internal derangements of knee.

- Arthroscopy should be reserved for selected cases where intervention is required and as a problem solving tool.

- $\quad$ MRI being easily available and non-invasive is useful as a pre-operative screening modality.

- Improves the quality of the arthroscopic evaluation and interventions and further reduces the morbidity.

\section{REFERENCES}

1. Gray SD, Kaplan PA, Dussalt RG. Imaging of knee: current status. ONCA 1997;28(4):643-58.
2. Kean DM, Worthington BS, Preston BJ, et al. Nuclear MRI of knee: examples of normal anatomy and pathology. Br J Radiology 1983;56(666):355-64.

3. Moon KL, Genant HK, Helms CA, et al. Musculoskeletal applications of nuclear MR. Radiology 1983;147(1):16171.

4. Li KC, Henkelman RM, Poon PY, et al. MR imaging of the knee. J Comput Assist Tomogra 1984;8(6):1147-54.

5. Hajek PC, Baker LL, Sartoris DJ, et al. Mr arthrography: an anatomic-pathologic investigation. Radiology 1987;163(1):141-7.

6. Kieser CW, Jackson RW. Severin nordentoft: the first arthroscopist. Arthroscopy 2001;17(5):532-5.

7. Bennett $\mathrm{CH}$, Chebli C. Knee Arthroscopy. Business Briefing North American Pharmacotherapy 2003;1-4.

8. Ruwe PA, Wright J, Randall RL, et al. Can MR imaging effectively replace diagnostic arthroscopy? Radiology 1992;183(1):335-9.

9. Elvenes J, Jerome $\mathrm{CP}$, Reikeras $\mathrm{O}$, et al. MRI as a screening procedure to avoid arthroscopy for meniscal tears. Arch Orthop Trauma Surg 2000;120(1-2):14-6.

10. Rappenport ED, Mehta S, Weislander SB, et al. MR imaging before arthroscopy in knee disorders? Acta Radiol 1996;37(3P2):602-9.

11. Kaplan PA, Gahl RH, Dussault RG, et al. Bone contusions of the posterior lip of the medial tibial plateau (contrecoup injuries) \& associated internal derangements of the knee at MR imaging. Radiology 1999;211(3):747-53.

12. Arndt WF, Traux AL, Barnett FM, et al. MR diagnosis of bone contusions of knee: comparison of coronal $\mathrm{T}_{2}$ weighted FSE with fat sat \& FSE STIR images with conventional STIR images. AJR 1996;166(1):119-24.

13. Sonin AH, Fitzgerald SW, Friedman H, et al. PCL injury: MR imaging diagnosis and pattern of injury. Radiology 1994;190(2):455-8.

14. Schweitzer ME, Tran D, Deely DM, et al. Medial collateral ligament injuries: evaluation of multiple signs, prevalence and location of associated bone bruises and assessment with MR imaging. Radiology 1995;194(3):825-9.

15. Yao L, Dungan D, Seeger LL. MR imaging of tibial collateral ligament injury: comparison with clinical examination. Skeletal Radiology 1994;23(7):521-4.

16. Levinson ME, Baker BE. Prearthrotomy diagnostic evaluation of the knee: review of 100 cases, diagnosed by arthrography and arthroscopy. AJR 1980;134(1):107-11.

17. Raunest J, Hotzinger H, Burrig KF. MRI and arthroscopy in detection of meniscal derangements. Arthroscopy 1994;10:624-30.

18. DeSmet AA, Norris MA, Yandow DR, et al. MR diagnosis of meniscal tears of the knee: importance of high signal in the meniscus that extends to the surface. American Journal of Roentgenology 1993;161:101-7. 